\title{
Preferência por comportamentos favoráveis à prática de atividade física e nível de atividade física de crianças de uma cidade do sul do Brasil
}

Preference for behavior conducive to physical activity and physical activity levels of children from a southern Brazil city

Programa de Pós-

Graduação em

Epidemiologia, Faculdade de Nutrição, Universidade Federal de Pelotas. R. Marechal Deodoro 1160, 3 andar. 96.020-220 Pelotas RS Brasil.

renatabielemann@

hotmail.com

${ }^{2}$ Faculdade de Nutrição.

Universidade Federal de Pelotas.
Abstract This article aims to describe preferences for behavior conducive to physical activity (PA) and to evaluate the influence of these preferences on physical activity of children from Pelotas in the state of Rio Grande do Sul. It involved a crosssectional study with children aged 4 to 11. Behavior conducive to PA was evaluated using the Netherlands Physical Activity Questionnaire (NPAQ). Time spent in moderate-to-vigorous physical activity (MVPA) was measured by accelerometry. Variance analysis and linear regression were performed to evaluate associations between questions from the NPAQ and independent variables and between each form of behavior and time spent in MVPA, respectively. Children in the higher economic bracket liked to draw more and preferred less vigorous games and playing outside than poorer children. Older children were less extrovert and liked to draw less than younger children. Enjoying sports, disliking drawing and liking to play outside were positively associated with daily time spent in MVPA. Some characteristics studied were associated with behavior conducive to $P A$, and economic status proved to be the most important influence. Preferences like enjoying sports positively influenced the time spent in MVPA.

Key words Physical activity, Behavior, Children, Cross-sectional study
Resumo O artigo objetiva descrever preferências por comportamentos favoráveis à prática de atividade física (AF) e avaliar sua influência sobre o nivel de AF de crianças da cidade de Pelotas, RS. Estudo transversal de base populacional com crianças de 4 a 11 anos. Comportamentos favoráveis à prática de AF foram avaliados pelo Netherlands Physical Activity Questionnaire (NPAQ). O tempo de AF moderada ou vigorosa (AFMV) foi medido por acelerometria. Análise de variância e regressão linear foram usadas para avaliar associações entre as perguntas do NPAQ e variáveis independentes e entre cada comportamento e o tempo de AFMV, respectivamente. Crianças de maior nível econômico gostaram mais de desenhar e preferiram menos brincadeiras agitadas e brincar na rua do que crianças de menor nível econômico. Crianças de maior idade foram menos extrovertidas e gostaram menos de desenhar do que crianças mais novas. Gostar de esportes, ter falta de interesse por desenhar e preferir brincar na rua foram positivamente associados ao tempo diário de AFMV. Algumas das características estudadas mostraram-se associadas a comportamentos favoráveis à prática de AF, sendo a maior influência observada para o nível econômico. Preferências como gostar de esportes influenciaram positivamente o tempo gasto em AFMV.

Palavras-chave Atividade física, Comportamentos, Crianças, Estudo transversal 


\section{Introdução}

Com o aumento da ocorrência de doenças crônicas não transmissíveis (DCNTs), cresceu também nas últimas décadas a preocupação com a prevenção de fatores de risco associados a elas, como a inatividade física ${ }^{1,2}$, a qual tem alta prevalência no Brasil ${ }^{3}$. A prática de atividade física é benéfica à saúde em todas as fases da vida e, devido ao fato das alterações metabólicas associadas ao surgimento de DCNTs serem observadas em idades cada vez mais precoces ${ }^{4,5}$, tem crescido a importância de estuda-las na infância, assim como seus determinantes.

A partir de estudos sobre o efeito tracking atualmente se sabe que crianças ativas apresentam maior chance de serem adolescentes ativos, que também têm maior probabilidade de serem adultos ativos ${ }^{6,7}$. Entretanto, além de considerar a importância do efeito tracking, também são relevantes diversos fatores associados à prática de atividade física nessas diferentes idades, pois determinantes individuais, econômicos, ambientais e sociais exercem influência sobre este comportamento. Um grupo de determinantes amplamente estudados, principalmente em adultos, é o das chamadas barreiras, entendidas como obstáculos percebidos pelo indivíduo que podem reduzir seu engajamento em com-portamentos saudáveis ${ }^{8}$. Dentre as percebidas pode-se citar, por exemplo, a falta de tempo ou dinheiro, a preguiça, a falta de companhia e até simplesmente o não gostar de se exercitar ${ }^{9,10}$. As barreiras percebidas pelos adolescentes e, mais especificamente pelas crianças, agregam-se àquelas observadas pelos seus pais devido à baixa autonomia que estes grupos apresentam na escolha das suas atividades. Assim, preocupação com temperatura, segurança, falta de alguém para levar também aparecem como barreiras nestas idades ${ }^{10}$.

As barreiras são percebidas em maior ou menor grau por diferentes grupos populacionais e manifestam-se pela prática de comportamentos desfavoráveis à prática de atividade física. No caso das crianças, podem se manifestar pela falta de interesse por brincar na rua e por atividades esportivas, ou inclusive por maior interesse por atividades calmas e dentro do domicílio, como desenhar e pintar. Mesmo com a dependência de seus pais apresentada por esta faixa etária, preferências por comportamentos favoráveis à maior prática de atividade física são importantes de serem consideradas para entendimento dos mecanismos que explicam o caminho pelo qual fatores psicossociais interferem na prática de atividade física das crianças.
O Netherlands Physical Activity Questionnaire é um questionário desenvolvido para avaliar a prática de atividade física em crianças. A capacidade de predição do nível de atividade física de crianças brasileiras foi considerada baixa em estudo recente, ainda que a correlação entre este instrumento e o padrão-ouro tenha sido similar ao apresentado por outros ${ }^{11}$. Embora com baixos valores de sensibilidade e especificidade para identificação da prática de atividade física, este questionário de sete questões é composto por seis perguntas que indicam preferências por comportamentos favoráveis à prática de atividade física, as quais podem variar entre as crianças, conforme as situações sociais, culturais e ambientais a que são expostas, o que pode levar a um maior entendimento das interações entre os determinantes distais e proximais da atividade física nessa idade.

Sendo assim, o objetivo do presente estudo é estudar a preferência por comportamentos favoráveis à prática de atividade física de crianças da cidade de Pelotas, RS, descrevendo-as conforme os fatores associados a estas preferências, bem como avaliar a associação entre estas preferências e o nível de atividade física avaliado de forma objetiva pelo método de acelerometria.

\section{Métodos}

Foi conduzido um estudo transversal de basepopulacional na cidade de Pelotas, uma cidade de médio porte localizada no sul do Brasil. A seleção amostral ocorreu por múltiplos estágios, sendo o primeiro o sorteio dos setores censitários, o segundo a seleção das residências e o terceiro a identificação dos indivíduos amostrados. Este estudo fez parte do Consórcio de Pesquisa do Mestrado em Epidemiologia da Universidade Federal de Pelotas.

Com base na divisão de setores censitários, disponibilizada pelo Instituto Brasileiro de Geografia e Estatística (IBGE) ${ }^{12}$, obtida no Censo de 2000, foram ordenados 400 deles considerados domiciliares da cidade conforme renda média dos chefes da família, de modo a garantir que setores dos mais diferentes níveis de renda fossem incluídos na seleção. Após, 130 setores foram sorteados com probabilidade igual ao tamanho, ou seja, considerando o número de domicílios em cada, que poderia ter aumentado ou diminuído desde o último recenseamento. Este processo de seleção garantiu que setores censitários com maior número de residências tivessem maior probabilidade de serem incluídos na seleção do que aque- 
les com menor número de domicílios. Todos os domicílios existentes em cada setor foram identificados e, a partir de sorteio sistemático, 10 domicílios foram selecionados em cada setor.

No primeiro contato da entrevistadora com as residências, todas as crianças que estavam com 4 a 10 anos de idade foram consideradas elegíveis e amostradas para o estudo. As entrevistadoras foram submetidas a um treinamento de 40 horas anteriormente à coleta de dados. As entrevistas foram conduzidas com as mães das crianças no próprio domicílio. No caso de não residência da mãe no mesmo domicílio de moradia da criança, as entrevistas foram realizadas com o principal cuidador. As entrevistas foram realizadas entre janeiro e maio de 2010

As preferências das crianças por comportamentos mais favoráveis à prática de atividade física foram medidas utilizando seis perguntas do Netherlands Physical Activity Questionnaire (NPAQ). Este questionário contém sete perguntas, sendo seis voltadas ao comportamento da criança e a última voltada à percepção da mãe sobre o nível de atividade física do filho, a qual não foi incluída neste estudo. As perguntas têm por base um período de referência de seis meses e são realizadas conforme este exemplo: "O $<$ nome da criança $>$ prefere brincar na rua ou dentro de casa?". As alternativas de resposta da mãe são de sempre, quase sempre ou tanto faz uma preferência ou a outra. A partir da resposta, pontuação em escala Likert de um a cinco é atribuída a cada pergunta, sendo que pontuação máxima é atribuída à resposta de "sempre", a alternativa mais favorável à prática de atividade física.

Demais variáveis coletadas na entrevista foram o sexo e idade da criança, a escolaridade e o nível de atividade física da mãe, obtido pela seção de lazer do International Physical Activity Questionnaire (IPAQ) $)^{13}$ - foram consideradas ativas as mães que atingiram a recomendação de pelo menos 150 minutos de atividade física por semana. Também foram perguntadas informações sobre o tipo de domicílio (casa ou apartamento), presença de outra criança de 4 a 11 anos na casa e nível econômico, obtido através de pontuação atribuída à presença de bens de consumo, empregada doméstica e escolaridade do chefe da família, conforme critério da Associação Brasileira de Empresas de Pesquisa (ABEP) do ano de $2010^{14}$.

De fevereiro a agosto do mesmo ano retornou-se aos domicílios das crianças cujas mães já haviam sido entrevistadas para entrega de monitores de atividade física (acelerômetros) da marca Actigraph modelo GT1M (LLC, Fort Walton Beach, FL, USA), cujas medidas são obtidas em counts.
Os acelerômetros foram utilizados pelas crianças por cinco dias, sendo entregues no sábado e devolvidos na quarta-feira seguinte. A captação dos movimentos pelo monitor foi programada para ocorrer a cada 5 segundos e as crianças foram recomendadas a utilizar o aparelho no quadril durante 24 horas, exceto durante o banho e outras atividades aquáticas, como a natação, por exemplo. Um supervisor de trabalho de campo realizou sistematicamente ligações aos domicílios em que constavam os aparelhos de forma a incentivar e checar o uso dos acelerômetros.

Os dados dos acelerômetros foram processados e analisados nos programas Actilife 4.4.1 e MAHUffe $^{15}$, respectivamente. O primeiro dia foi excluído da análise, devido à possível mudança de comportamento. Foram excluídos das análises períodos do dia com mais de 10 minutos consecutivos de zero counts e dias em que a utilização do aparelho foi inferior a 600 minutos. O tempo de atividade física moderada ou vigorosa foi obtido pela média de minutos por dia em que as atividades executadas pelas crianças foram de intensidade de pelo menos 2000 counts por minuto em sessões de no mínimo dez minutos de duração, o que corresponde em crianças, à caminhada em velocidade de em torno de $3 \mathrm{~km} / \mathrm{h}^{16}$.

Além da descrição das características sociodemográficas das crianças estudadas, também são mostradas as proporções de resposta para cada pontuação nas perguntas pertencentes ao NPAQ. A análise bruta foi realizada entre as seguintes variáveis independentes: sexo, idade, nível econômico, escolaridade materna, atividade física materna, tipo de domicílio e presença de outra criança da mesma faixa etária em casa e a pontuação de cada questão proveniente do NPAQ (variando de 1 a 5 pontos) utilizando análise de variância (ANOVA) ou teste de Kruskal-Wallis, conforme presença ou não de heterogeneidade da variância entre os estratos de exposição. Além disso, regressão linear múltipla foi conduzida entre a pontuação em cada uma das perguntas do NPAQ e o tempo gasto em atividade física moderada ou vigorosa avaliado pelo acelerômetro, incluindo-se todas as outras variáveis como possíveis confundidoras no modelo quando valor $\mathrm{p}<0,20$ na análise bruta com o desfecho. $\mathrm{O}$ efeito de delineamento foi considerado em todas as análises estatísticas. Foram consideradas significativas as associações cujo $\mathrm{p}<0,05$.

O estudo foi aprovado pelo Comitê de Ética em Pesquisa da Faculdade de Medicina da Universidade Federal de Pelotas. Todas as mães assinaram termo consentimento livre e esclarecido anteriormente à realização das entrevistas. 


\section{Resultados}

O processo de seleção amostral identificou 379 crianças, das quais 369 tiveram suas mães entrevistadas (97,4\% de resposta). Entretanto, apenas 239 crianças forneceram dados válidos na acelerometria. A Tabela 1 mostra a distribuição das características destas crianças. Pouco mais da metade (52\%) das crianças foi do sexo masculino e a menor parte delas estava com 10 ou 11 anos de idade no momento da entrevista (18,5\%). Pouco mais de $10 \%$ das crianças pertencia às classes econômicas mais altas (A/B) e a maioria da amostra foi composta por filhos de mães com no máximo ensino fundamental completo. Mais de $80 \%$ destas crianças foram filhos de mães inativas, a maior parte mora em casa e não vive com outra criança de mesma faixa etária. Pouco mais de um quarto das crianças não atinge a reco-

Tabela 1. Características demográficas, socioeconômicas e comportamentais das crianças e mães de amostra populacional da cidade de Pelotas, RS, 2010.

\begin{tabular}{|c|c|}
\hline Variáveis & $\mathrm{N}(\%)$ \\
\hline \multicolumn{2}{|l|}{ Sexo } \\
\hline Masculino & $192(52,0)$ \\
\hline Feminino & $177(48,0)$ \\
\hline \multicolumn{2}{|l|}{ Idade } \\
\hline 4-5 anos & $103(27,9)$ \\
\hline $6-7$ anos & $89(24,1)$ \\
\hline $8-9$ anos & $109(29,5)$ \\
\hline 10-11 anos & $68(18,5)$ \\
\hline \multicolumn{2}{|l|}{ Nível econômico (ABEP) } \\
\hline $\mathrm{A} / \mathrm{B}$ & $44(11,9)$ \\
\hline $\mathrm{C}$ & $167(45,3)$ \\
\hline $\mathrm{D} / \mathrm{E}$ & $158(42,8)$ \\
\hline \multicolumn{2}{|c|}{ Escolaridade materna (anos de estudo) } \\
\hline $0-4$ anos & $64(17,4)$ \\
\hline $5-8$ anos & $150(40,7)$ \\
\hline $9-11$ anos & $107(29,1)$ \\
\hline 12 anos ou mais & $47(12,8)$ \\
\hline \multicolumn{2}{|c|}{ Atividade física materna ( $\geq 150 \mathrm{~min} / \mathrm{sem})$} \\
\hline Sim & $65(18,3)$ \\
\hline Não & $291(81,7)$ \\
\hline \multicolumn{2}{|l|}{ Tipo de domicílio } \\
\hline Casa & $322(87,3)$ \\
\hline Apartamento & $47(12,7)$ \\
\hline \multicolumn{2}{|c|}{ Outra criança de 4-11 anos no domicílio } \\
\hline Sim & $159(43,1)$ \\
\hline Não & $210(56,9)$ \\
\hline \multicolumn{2}{|c|}{ Atividade física ${ }^{*}(\geq 300 \mathrm{~min} / \mathrm{sem}$ ) } \\
\hline Sim & $175(73,2)$ \\
\hline Não & $64(26,8)$ \\
\hline
\end{tabular}

mendação atual de prática de atividade física ( $\geq$ $60 \mathrm{~min} / \mathrm{dia})$.

As preferências de comportamento das crianças são mostradas na Tabela 2. Em torno de $70 \%$ das crianças quase sempre ou sempre preferem brincar com outras crianças e são extrovertidas. Vinte por cento delas quase sempre ou sempre preferem brincadeiras calmas e quase $90 \%$ quase sempre ou sempre gostam de praticar esportes. Em torno de 20\% das crianças quase sempre ou sempre não se interessam por desenhar, pintar ou ver revistas e aproximadamente $60 \%$ delas quase sempre ou sempre preferem brincar na rua ou no pátio.

A Tabela 3 mostra a associação entre as características estudadas e a pontuação obtida em cada comportamento perguntado no NPAQ, de forma que grupos com maiores médias apresentam preferências por comportamentos mais favoráveis à prática de atividade física. Nenhuma das variáveis independentes estudadas foi estatisticamente associada com maior pontuação com relação à preferência por brincar com outras crianças. Já com relação à preferência por brincadeiras agitadas ou calmas, crianças provenientes de famílias de menor nível econômico ( $\mathrm{p}$ $=0,004)$ e filhos de mães de menor escolaridade $(\mathrm{p}=0,001)$ apresentaram maior pontuação na preferência por brincadeiras agitadas do que crianças de maior nível econômico e que cujas mães estudaram por mais tempo. Apenas a idade foi estatisticamente associada à pontuação relacionada a gostar de praticar esportes, pois crianças de 8 e 9 anos apresentaram média de 4,60 (dp = $0,86)$ nesta questão, a qual foi superior à média obtida nos outros grupos de idade $(\mathrm{p}=0,028)$. Quanto à extroversão ou introversão, Crianças mais novas foram relatadas como mais extrovertidas pelas suas mães $(p=0,008)$, assim como crianças que não moram com outra criança de mesma faixa etária foram consideradas mais extrovertidas do que aquelas crianças que vivem com outra criança também de 4 a 11 anos em casa $(p=0,020)$. Meninos apresentaram maior pontuação quanto à falta de interesse por desenhar, pintar ou ver revistas do que meninas ( $\mathrm{p}=$ 0,002), bem como também crianças de maior idade também manifestaram falta de interesse por estas atividades $(p=0,009)$. Crianças de menor nível econômico e filhos de mães de menor escolaridade também apresentaram maior pontuação em falta de interesse por desenhar, pintar ou ver revistas $(\mathrm{p}=0,012 ; \mathrm{p}=0,008)$. Maior escore na falta de interesse por tais atividades também foi observada em crianças que moram com outras crianças de mesma faixa etá- 
Tabela 2. Prevalência de comportamentos relacionados à prática de atividade física de crianças de 4 a 11 anos moradoras da cidade de Pelotas, RS. 2010.

\begin{tabular}{|c|c|c|c|c|}
\hline & $\begin{array}{l}\text { Sempre ou } \\
\text { Quase sempre } \\
\quad(1 \text { ou } 2) \\
\mathrm{N}(\%)\end{array}$ & $\begin{array}{l}\text { Tanto } \\
\text { faz } \\
(3) \\
\mathrm{N}(\%)\end{array}$ & $\begin{array}{l}\text { Quase sempre } \\
\text { ou Sempre } \\
\text { (4 ou } 5) \\
\mathrm{N}(\%)\end{array}$ & \\
\hline Preferem brincar sozinho & $63(17,1)$ & $42(11,4)$ & $264(71,5)$ & $\begin{array}{l}\text { Preferem brincar com outras } \\
\text { crianças }\end{array}$ \\
\hline $\begin{array}{l}\text { Preferem brincadeiras } \\
\text { calmas, como quebra-cabeça, } \\
\text { cartas, massinha e brinquedos } \\
\text { de encaixar }\end{array}$ & $81(21,9)$ & $53(14,4)$ & $235(63,7)$ & $\begin{array}{l}\text { Preferem brincadeiras } \\
\text { agitadas, como correr, subir } \\
\text { em coisas, lutar, saltar e } \\
\text { pular corda }\end{array}$ \\
\hline $\begin{array}{l}\text { Não gostam de praticar } \\
\text { esportes }\end{array}$ & $33(8,9)$ & $13(3,5)$ & $323(87,5)$ & $\begin{array}{l}\text { Gostam de praticar esportes, } \\
\text { como jogar bola e andar de } \\
\text { bicicleta }\end{array}$ \\
\hline $\begin{array}{l}\text { São mais introvertidos, quietos } \\
\text { e gostam de ficar em casa }\end{array}$ & $77(20,9)$ & $22(5,9)$ & $270(73,2)$ & $\begin{array}{l}\text { São mais extrovertidos, } \\
\text { gostam de sair }\end{array}$ \\
\hline $\begin{array}{l}\text { Gostam de desenhar, pintar } \\
\text { ou ver revistas }\end{array}$ & $278(75,3)$ & $24(6,5)$ & $67(18,2)$ & $\begin{array}{l}\text { Não se interessam por } \\
\text { desenhar, pintar ou ver } \\
\text { revistas }\end{array}$ \\
\hline $\begin{array}{l}\text { Preferem brincar dentro de } \\
\text { casa ou da escola }\end{array}$ & $85(23,0)$ & $67(18,2)$ & $217(58,8)$ & $\begin{array}{l}\text { Preferem brincar na rua, no } \\
\text { pátio }\end{array}$ \\
\hline
\end{tabular}

Fuente: Elaboración propia a partir de datos del Sistema Nacional de Información en Salud (SINAIS), el Instituto Nacional de Estadística Geografía e Informática (INEGI) y el Consejo Nacional de Población (CONAPO)

ria $(\mathrm{p}=0,028)$. Quanto à preferência por brincar na rua ou dentro de casa, crianças de menor nível econômico apresentaram maior escore na preferência por brincar da rua do que crianças de maior nível econômico $(\mathrm{p}=0,008)$. Da mesma forma, crianças que vivem com outras crianças de mesma faixa etária também apresentaram maior preferência por brincar na rua ou no pátio do que as demais $(p=0,040)$.

A influência de cada um destes comportamentos sobre a prática de atividade física moderada ou vigorosa, medida objetivamente pela acelerometria, e após ajuste para possíveis confundidores, é apresentada na Figura 1. Embora todas as questões tenham apresentado coeficientes de regressão positivos com o aumento da pontuação, não foi encontrada associação estatisticamente significativa entre preferência por brincadeiras agitadas ou com outras crianças e o tempo médio gasto por dia com atividade física de intensidade pelo menos moderada. Também não foi encontrada associação entre maior extroversão e o tempo gasto em atividade física destas intensidades. Em contrapartida, cada ponto a mais na questão sobre preferência por brincadeira na rua ou no pátio foi estatisticamente associado com em torno de 3,4 minutos a mais de atividade física moderada ou vigorosa por dia $(p=0,006)$. Da mesma forma, um ponto a mais em direção à criança sempre manifestar falta de interesse por desenhar, pintar ou ver revistas, aumenta em média a prática de atividade física moderada ou vigorosa em 2,8 minutos por dia $(\mathrm{p}=0,028)$. Ainda, um ponto a mais em direção à criança sempre gostar de praticar esportes adiciona em média 4,1 minutos de atividade física de intensidade pelo menos moderada por dia $(\mathrm{p}$ $=0,007$ ), o que significa que crianças que sempre gostam de praticar esportes (5 pontos) gastam em torno de 16 minutos a mais por dia em atividade física moderada ou vigorosa do que aquelas crianças que sempre não gostam de praticar esportes (1 ponto). Além disso, após inserir todas as perguntas no modelo foi visto que o coeficiente de todas as questões se mantém praticamente iguais, de modo que o efeito de cada preferência não desaparece após ser levado em consideração o efeito de outra.

\section{Discussão}

O presente estudo mostrou associações entre características sociodemográficas e comportamentais de mães e crianças e preferências por comportamentos favoráveis à prática de atividade física entre crianças de 4 a 11 anos, além de observar a influência de cada uma dessas prefe- 


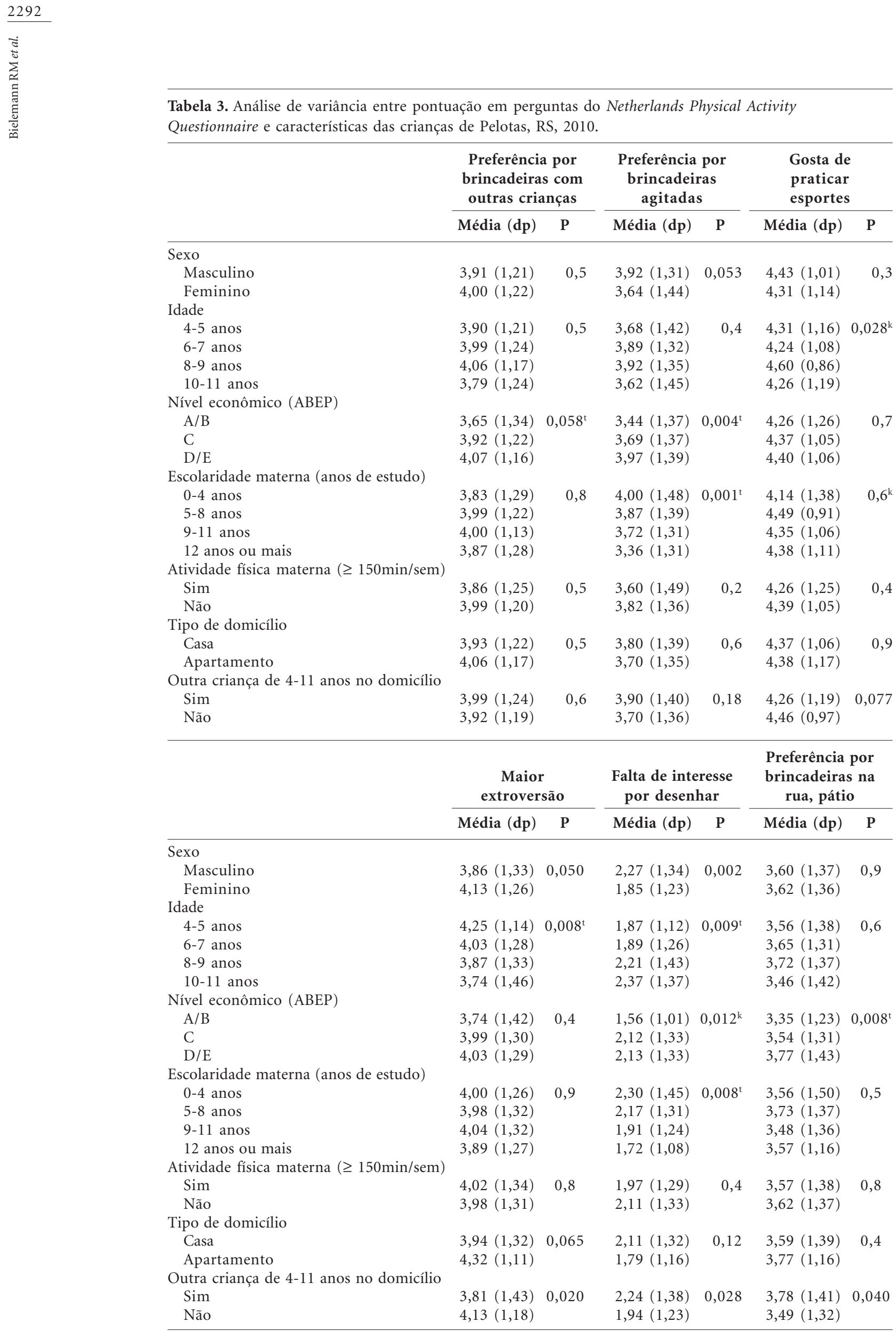

${ }^{\mathrm{k}}$ Teste de Kruskal-Wallis; ${ }^{\mathrm{t}}$ Teste de tendência linear. 


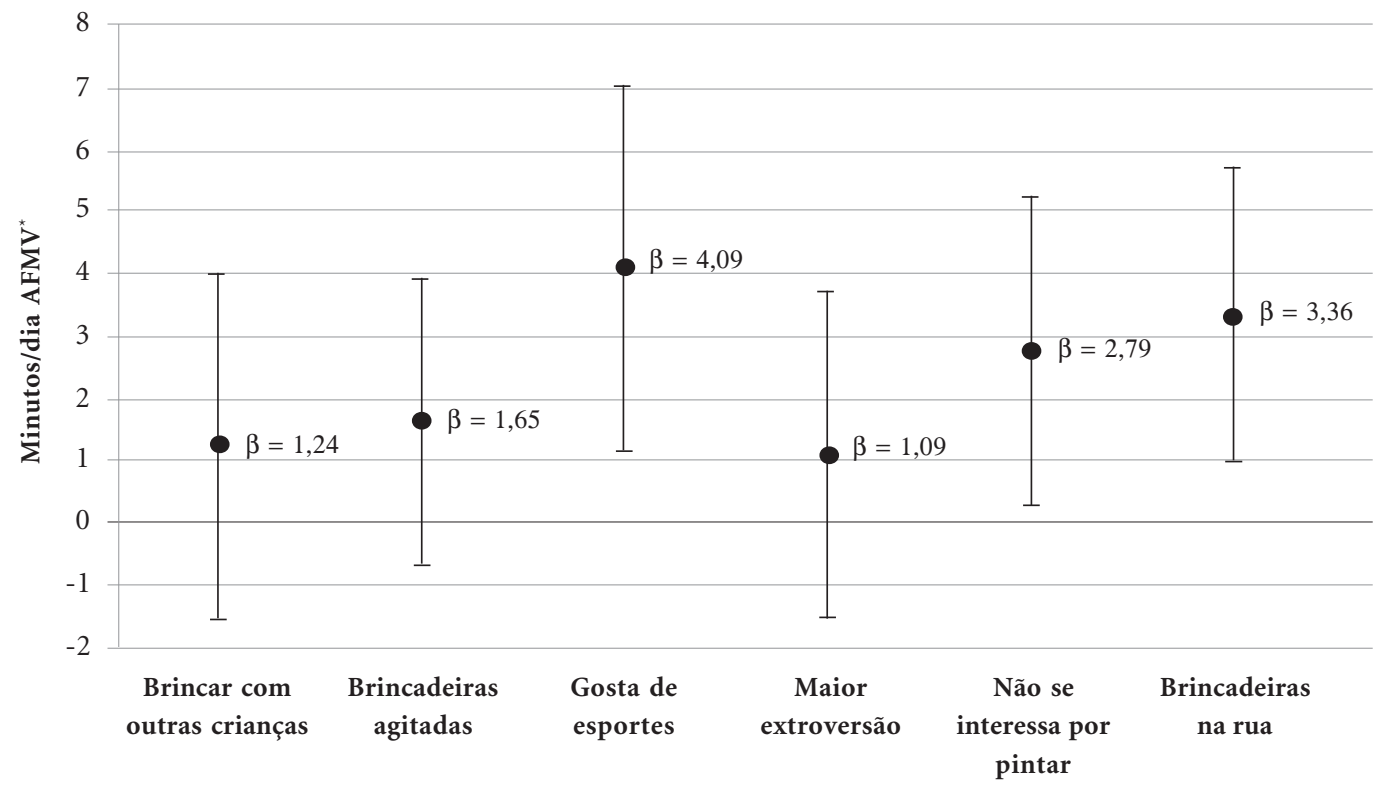

Figura 1. Regressão linear múltipla entre pontuação obtida em cada pergunta do Netherlands Physical Activity Questionnaire e tempo diário de práticas de atividade física moderada ou vigorosa de crianças de 4 a 11 residentes na zona urbana do município de Pelotas, RS, 2010.

AFMV - Atividade física moderada ou vigorosa; " Coeficiente de regressão $(\beta)$ ajustado para as variáveis: sexo, idade, nível econômico, tipo de domicílio, presença de outra criança de 4 a 11 anos na residência e atividade física materna. $(\mathrm{N}=231)$

rências, percebidas pelas mães, sobre o tempo gasto em atividade física de intensidade moderada ou vigorosa. Mais de 70\% das crianças atingiu a recomendação atual de prática de atividade física para esta faixa etária. Pelo menos $60 \%$ das crianças do estudo preferiram habitualmente quase sempre ou sempre comportamentos favoráveis à prática de atividade física, com exceção de apenas $18 \%$ que quase sempre ou sempre não se interessavam por desenhar, pintar ou ver revistas. Quanto aos fatores associados a estas preferências, foi visto que a idade e o nível econômico foram as características estudadas mais associadas a elas, de forma que crianças mais velhas são mais introvertidas e preferem menos desenhar do que crianças mais novas. Já crianças de menor status econômico estatisticamente preferiram mais brincadeiras agitadas, apresentaram maior falta de interesse por desenhar e preferiram mais brincar na rua em comparação a crianças de maior nível econômico. Todas essas preferências apresentaram influência positiva na prática de atividade física de intensidade no mínimo moderada. Entretanto, apenas o gostar de esportes, a falta de interesse por desenhar, pintar ou ver revistas e a preferência por brincar na rua foram estatisticamente associadas ao tempo gasto em atividade física moderada ou vigorosa.

A principal força deste estudo está na amostragem, a qual garantiu equiprobabilidade na seleção das crianças incluídas na amostra e também no uso de acelerômetros para avaliação da atividade física, uma vez que o uso de questionários para este fim apresenta diversas desvantagens nesta faixa etária ${ }^{17}$. Além disso, as perdas na primeira fase do estudo foram de apenas 2,6\%. Em contrapartida, informações da acelerometria não foram disponíveis para em torno de $35 \%$ da amostra devido à utilização por tempo menor do que o necessário ou recusa. Entretanto, a amostra que utilizou os acelerômetros $(\mathrm{N}=239)$ foi diferente do restante somente em relação à escolaridade materna, pois crianças filhos de mães de escolaridade intermediária (grupos de 5 a 8 e de 9 a 11 anos completos de estudo) foram os com menor percentual de dados válidos na acelerometria. Caso as perdas fossem distribuídas igualmente conforme os grupos de escolaridade materna, poderíamos ter encontrado média de pontuação superior à preferência por brincadeiras 
agitadas e falta de interesse em desenhar, uma vez que estes comportamentos foram associados positivamente à escolaridade materna. Outra limitação consistiu em relação à falta de poder estatístico para condução das análises uma vez que foi menor do que $80 \%$ para todas as associações avaliadas. Isso pode ter interferido para que algumas associações nas quais poderiam aparecer em um tamanho de amostra maior não fossem verificadas neste estudo. Porém, de certa forma este fato reforça as associações já encontradas.

Percentual elevado das crianças da amostra preferiu quase sempre ou sempre comportamentos mais favoráveis à prática de atividade física. Entretanto, como esperado, este padrão foi variável conforme o comportamento e as características de exposição avaliadas. Avaliação da prática de atividade física pela acelerometria e fatores associados na mesma amostra encontrou que as crianças de menor nível econômico são mais ativas do que as crianças de maior ${ }^{18}$. A preferência por brincadeiras agitadas, maior falta de interesse por desenhar, pintar ou ver revistas e também a preferência por atividades fora de casa ou escola pelas crianças de menor nível econômico encontrada neste estudo poderia ajudar a explicar os mecanismos envolvidos na diferença do tempo de atividade física moderada ou vigorosa entre esses grupos.

A maior falta de interesse por desenhar, pintar ou ver revistas em crianças de menor nível econômico e filhos de mães de menor escolaridade pode ser explicada por estudo conduzido com a coorte de nascimentos de 2004 da mesma cidade no qual foi encontrado que crianças filhos de mães de menor escolaridade são menos estimuladas a fatores que favorecem o desenvolvimento cognitivo como ter um livro e ouvir histórias $^{19}$, os quais podem de alguma forma estar associados ao hábito de desenhar, pintar ou ver revistas. Não encontramos explicação na literatura para o fato de crianças de menor nível econômico serem mais extrovertidas e, diferente das outras preferências avaliadas que foram associadas ao nível econômico, a extroversão não foi associada à escolaridade da mãe.

Quanto à maior preferência por brincar na rua ou no pátio apresentada por crianças de menor nível econômico, é possível que pelo menos parte desta associação possa ser explicada por fatores relacionados ao ambiente social, como a segurança. Isso pode se dar pelo fato de que a percepção de falta de segurança do bairro, a qual é associada com a menor frequência de brincadeiras em espaços ao ar livre ${ }^{20}$, é mais observada entre mães de crianças de maior nível econômico e escolaridade ${ }^{21}$.
$\mathrm{O}$ ambiente físico da área externa da residência, escola e também da vizinhança influencia a prática de atividade física de crianças e a preferência por brincadeiras ao ar livre ${ }^{22}$. Ambiente físico favorável é visto em locais de moradia de famílias de maior nível econômico ${ }^{23,24}$. Sendo assim, seria de se esperar que a preferência por atividades na área externa da casa ou da escola fosse encontrada entre crianças de maior nível econômico, o que não foi observado no presente estudo.

A prática de atividade física dos pais também é um fator encontrado na literatura como associado com a prática de atividade física de crianças, de forma que os filhos de pais ativos praticam mais atividade física do que os de pais inati$\operatorname{vos}^{25,26}$. Na literatura é apontado que esta influência pode dar-se em parte pelo ambiente social, expresso pelo incentivo e suporte social dos pais para a prática de atividades de esporte e lazer ativo dos filhos, pois pais que gastam maior tempo praticando atividade física podem incentivar mais os seus filhos para o envolvimento com atividades físicas e ter o apoio da família tem sido associado com aumento da AF entre os joven ${ }^{27}$. Porém, no presente estudo esta hipótese não foi evidenciada, uma vez que a prática de atividade física das mães dessas crianças não foi associada com nenhuma das preferências de comportamento favoráveis à prática de atividade física.

A presença de irmãos no domicílio tem sido associada com a prática de atividade física de crianças em diversos estudos ${ }^{28,29}$, pois a companhia em brincadeiras, motivação e oportunidades para a prática de atividades não sedentárias é maior com o convívio com crianças de mesma faixa etária. No presente estudo não existe a informação específica sobre a moradia com irmãos, mas há a informação sobre a presença de outra criança da mesma faixa etária da população alvo que na maioria dos casos pode ser irmã/irmão, mas também poderia ser um primo, por exemplo. A presença de outra criança da faixa etária em estudo foi associada à maior falta de interesse por desenhar e também por preferência maior por brincar na rua ou no pátio de casa ou da escola, o que de certa forma reforça que o fato da presença de uma companhia de idade semelhante é importante para o desenvolvimento de um ambiente social favorável à prática de atividades que diminuam a exposição ao lazer sedentário, criando situações propícias ao envolvimento com atividades mais intensas ${ }^{27,29}$.

Embora a maior falta de interesse por desenhar, pintar ou ver revistas tenha sido associada ao maior tempo gasto por essas crianças com atividades moderadas ou vigorosas, deve-se ter 
cautela ao explicar este dado para mães, professores ou gestores, uma vez que essas atividades são importantes para o desenvolvimento cognitivo dessa faixa etária. As crianças estão em uma fase de descoberta de imagens, cores e palavras e privá-las dessas atividades pode causar danos em seu desenvolvimento, uma vez que a leitura nesta idade influencia o rendimento escolar e a melhoria do vocabulário - podendo inclusive ser mais importante do que o ensino formal e a linguagem oral ${ }^{30}$, além de que atividades como desenhar e pintar são importantes para o desenvolvimento da coordenação motora fina ${ }^{31}$.

Os maiores coeficientes entre a pontuação das perguntas e o tempo de atividade física moderada ou vigorosa foram observados para a preferência por brincadeiras na rua e para gostar de esportes. As crianças que gastam um maior tempo em brincadeiras ao ar livre são mais ativas do que aquelas que executam mais atividades do tempo livre no ambiente doméstico. Neste sentido, faz-se necessária a tentativa de se resgatar o hábito do brincar de maneira não estruturada na rua, um comportamento que foi reduzido nos últimos anos devido à competitividade com aqueles chamados de sedentários, como assistir televisão, usar o computador e jogar vídeo game, atividades muitas vezes consideradas "mais seguras" pelos pais ${ }^{21}$. Este fato é reforçado ainda pela questão que não somente maiores níveis de atividade física são observados entre crianças que brincam ao ar livre, mas também maior bem estar, interação social e inteligência emocional são promovidos pelo hábito de brincar livremente na rua ${ }^{21}$.

\section{Colaboradores}

RM Bielemann, MO Xavier e DP Gigante participaram igualmente de todas as etapas de elaboração do artigo.
Além disso, a diferença média de 16 minutos a mais de prática de atividade física moderada ou vigorosa encontrada entre crianças que sempre gostam de esportes e aquelas que sempre não gostam de esportes chama a atenção para a importância que a prática esportiva tem na promoção da atividade física no período da infância. Este achado é de grande valia uma vez que as atividades esportivas tendem a ser mais intensas que algumas atividades não estruturadas, além de promover socialização, disciplina e habilidades motoras específicas; aspectos importantes para este período da vida e que incentivam a manutenção da prática esportiva e de outras atividades físicas em geral em períodos posteriores da vida ${ }^{32-34}$.

Os resultados deste estudo mostraram a importância que as características sociodemográficas, principalmente o nível econômico e a idade, têm sobre a preferência por comportamentos favoráveis à prática de atividade física de crianças. Além disso, também foi observada a influência que algumas dessas preferências apresentaram sobre o tempo gasto em atividade física de intensidade moderada ou vigorosa, sendo que gostar de esportes foi a preferência avaliada pelas questões do NPAQ que mais influenciou o nível de atividade física destas crianças. As crianças devem ser amplamente estimuladas no ambiente físico e social de modo a serem capazes de optarem por comportamentos estimuladores da atividade física sempre que possível, diminuindo as barreiras sociodemográficas existentes entre as ativas e as inativas. 
4. Daniels SR, Greer FR. Lipid screening and cardiovascular health in childhood. Pediatrics 2008; 122(1):198-208.

5. Halfon N, Verhoef PA, Kuo AA. Childhood antecedents to adult cardiovascular disease. Pediatr Rev 2012; 33(2):51-60.

6. Azevedo MR, Araujo CL, Cozzensa da Silva M, Hallal PC. Tracking of physical activity from adolescence to adulthood: a population-based study. Rev Saude Publica 2007; 41(1):69-75.

7. Kjonniksen L, Torsheim T, Wold B. Tracking of leisure-time physical activity during adolescence and young adulthood: a 10-year longitudinal study. Int J Behav Nutr Phys Act 2008; 5:69.

8. Brown SA. Measuring perceived benefits and perceived barriers for physical activity. Am J Health Behav 2005; 29(2):107-116.

9. Reichert FF, Barros AJ, Domingues MR, Hallal PC. The role of perceived personal barriers to engagement in leisure-time physical activity. Am J Public Health 2007; 97(3):515-519.

10. Santos MS, Hino AA, Reis RS, Rodriguez-Anez CR Prevalence of barriers for physical activity in adolescents. Rev Bras Epidemiol 2010; 13(1):94-104.

11. Bielemann RM, Reichert FF, Paniz VM, Gigante DP. Validation of the Netherlands physical activity questionnaire in Brazilian children. Int J Behav Nutr Phys Act 2011; 8:45.

12. Instituto Brasileiro de Geografia e Estatística (IBGE). Censo de 2000. Rio de Janeiro: IBGE; 2000.

13. Craig CL, Marshall AL, Sjostrom M, Bauman AE Booth ML, Ainsworth BE, Pratt M, Ekelund U, Yngve A, Sallis JF, Oja P. International physical activity questionnaire: 12 -country reliability and validity. Med Sci Sports Exerc 2003; 35(8):1381-1395.

14. Associação Brasileira de Empresas de Pesquisa (ABEP). Critério de Classificação Econômica Brasil. São Paulo: ABEP; 2010.

15. MAHUffe. [computer program]. Cambridge: University of Cambridge School of Clinical Medicine. [acessado ano mês dia]. www.mrc-epid.cam.ac.uk [completar esta referência]

16. Ekelund U, Sardinha LB, Anderssen SA, Harro M Franks PW, Brage S, Cooper AR, Andersen LB, Riddoch C, Froberg K. Associations between objectively assessed physical activity and indicators of body fatness in 9- to 10-y-old European children: a population-based study from 4 distinct regions in Europe (the European Youth Heart Study). Am J Clin Nutr 2004;80(3):584-590.

17. Welk GJ, Corbin CB, Dale D. Measurement issues in the assessment of physical activity in children. Res Q Exerc Sport 2000; 71(Supl. 2):S59-73.

18. Bielemann R, Cascaes A, Reichert F, Domingues $\mathrm{M}$, Gigante D. Objectively measured physical activity in children from a Southern Brazilian city: a population-based study. J Phys Act Health 2013; 10(8):1145-1152.

19. Barros AJ, Matijasevich A, Santos IS, Halpern R. Child development in a birth cohort: effect of child stimulation is stronger in less educated mothers. Int J Epidemiol 2010; 39(1):285-294.

20. Duke NN, Borowsky IW, Pettingell SL. Parent perceptions of neighborhood: relationships with US youth physical activity and weight status. Matern Child Health I 2012; 16(1):149-157.
21. Burdette HL, Whitaker RC. A national study of neighborhood safety, outdoor play, television viewing, and obesity in preschool children. Pediatrics 2005; 116(3):657-662.

22. Franzini L, Elliott MN, Cuccaro P, Schuster M, Gilliland MJ, Grunbaum JA, Franklin F, Tortolero SR. Influences of physical and social neighborhood environments on children's physical activity and obesity. Am J Public Health 2009; 99(2):271-278.

23. Trasande L, Cronk C, Durkin M, Weiss M, Schoeller D, Gall E, Hewitt J, Carrel A, Landrigan P, Gillman $M$. Environment and obesity in the National Children's Study. Cien Saude Colet 2010; 15(1):195-210.

24. Tandon PS, Zhou C, Sallis JF, Cain KL, Frank LD, Saelens BE. Home environment relationships with children's physical activity, sedentary time, and screen time by socioeconomic status. Int J Behav Nutr Phys Act 2012; 9:88.

25. Fuemmeler BF, Anderson CB, Masse LC. Parentchild relationship of directly measured physical activity. Int J Behav Nutr Phys Act 2011; 8:17.

26. Kohl HW 3rd, Hobbs KE. Development of physical activity behaviors among children and adolescents. Pediatrics 1998; 101(3 Pt 2):549-554.

27. Duncan SC, Duncan TE, Strycker LA, Chaumeton NR. A multilevel approach to youth physical activity research. Exerc Sport Sci Rev 2004; 32(3):95-99.

28. McMinn AM, van Sluijs EM, Nightingale CM, Griffin SJ, Cook DG, Owen CG, Rudnicka AR, Whincup $\mathrm{PH}$. Family and home correlates of children's physical activity in a multi-ethnic population: the crosssectional Child Heart and Health Study in England (CHASE). Int J Behav Nutr Phys Act 2011; 8:11.

29. Katzmarzyk PT, Baur LA, Blair SN, Lambert EV, Oppert JM, Riddoch C. International conference on physical activity and obesity in children: summary statement and recommendations. Int J Pediatr Obes 2008; 3(1):3-21.

30. Cunningham AE, Stanovich KE. What reading does for the mind. Journal of Direct Instruction 2001 1(2):137-149.

31. Haydari A, Askari P, Nezhad MZ. Relationship between affordances in the home environment and motor development in children age 18-42 months. Journal of Social Sciences 2009; 5(4):319-328.

32. Kjonniksen L, Anderssen N, Wold B. Organized youth sport as a predictor of physical activity in adulthood. Scand J Med Sci Sports 2009; 19(5):646-654.

33. Kuh D, Cooper C. Physical activity at 36 years: patterns and childhood predictors in a longitudinal study. J Epidemiol Community Health 1992; 46(2): 114-119.

34. Richards R, Williams S, Poulton R, Reeder AI. Tracking club sport participation from childhood to early adulthood. Res Q Exerc Sport 2007; 78(5):413-419.

Artigo apresentado em 07/05/2013

Aprovado em 27/06/2013

Versão final apresentada em 08/07/2013 